\title{
Saturation of the coherent beam-beam instability
}

\author{
S. Heifets \\ Stanford Linear Accelerator Center, Stanford University, Stanford, California 94309
}

(Received 22 December 2000; published 12 April 2001)

\begin{abstract}
The nonlinear regime of the beam-beam instability for flat beams is considered. Excitation of coherent modes and mode interaction is studied above the threshold of instability. It is shown that the exponential growth of the linear approximation may saturate, leading to a finite growth of transverse emittance.
\end{abstract}

DOI: $10.1103 /$ PhysRevSTAB.4.044401

PACS numbers: 29.27.Bd

\section{INTRODUCTION}

The beam blowup due to the beam-beam interaction is one of the main factors limiting luminosity and lifetime in circular colliders. This effect is well known and has been studied theoretically, numerically, and experimentally. In particular, the coherent beam-beam instability was studied and its importance was emphasized; see, for example, [1-5].

This paper considers two mechanisms of beam-beam blowup: one is due to harmonics of periodic beam-beam kicks generated by a rigid bunch of the opposite beam, and the second mechanism is due to saturation of the linearly unstable beam-beam coherent modes. The latter is similar to the microwave instability. This mechanism can be described as excitation of unstable modes which, in their turn, modify the bunch distribution to a new steady-state equilibrium. We hope these mechanisms may explain emittance blowup in the flip-flop regime. A flat beam is assumed with parameters of the PEP-II B Factory [6]. For the sake of completeness, the basic formulas are rederived.

This paper is organized as follows: In the next section, the notations are introduced and the explicit form of the beam-beam potential and tune shift are given for a flat beam. Emittance growth due to beam-beam resonances for rigid bunches is estimated in Sec. III. In Sec. IV, we consider a nonlinear resonance, which selects two coupled resonance azimuthal modes. The Vlasov equations for the selected azimuthal modes are derived and solved in Sec. V in the linear approximation. The solution describes the radial modes and allows analysis of their stability. The radial modes, independent in the linear approximation, interact due to coupling between modes. The mode interaction is considered in Sec. VI in quasilinear theory. Saturation of a single linearly unstable radial mode is described in Sec. VII. Interaction of two radial modes is analyzed in Sec. VIII. The results are summarized in Sec. IX.

\section{BASIC KINEMATICS AND NOTATIONS}

Beam-beam interaction of flat beams can be described by 1D model averaging the Coulomb interaction (doubled to take into account the magnetic field force) over Gaussian distribution in the horizontal plane. The steady state can be described by the Hamiltonian averaged over the revolution period. Below the threshold of coherent instability, action-angle variables $I, \alpha$ can be chosen to make the steady-state (Haissinski) Hamiltonian $H_{H}(I)$ independent of $\alpha$. In the lowest order in the beam-beam parameter, we can neglect all anharmonic terms of particle motion. In this approximation, the vertical position of a particle $y=\Delta y_{0}+w \sqrt{2 I \epsilon_{y}} \cos \alpha$, where $\epsilon=\sigma^{2} / \beta$ is vertical beam emittance, $2 \Delta y_{0}$ is distance between beam centroids (vertical beam offset), $w=\sqrt{\beta_{y}}$, and $\beta_{y}$ is vertical beta function.

The steady-state normalized distribution function $\rho_{H}(I)$ of a beam also depends only on $I, \rho_{H}(I)=\frac{1}{2 \pi} e^{-I}$. Distribution $\rho_{H}$ is normalized, $2 \pi \int d I \rho_{H}(I)=1$.

The full distribution function $\rho(I, \alpha, \phi)$ is the sum of azimuthal harmonics. For the first bunch,

$$
\begin{aligned}
\rho_{1}(I, \alpha, \phi) & =\rho_{H}^{(1)}(I)+\sum_{n=-\infty}^{\infty} \rho_{n}^{(1)}(I, \phi) e^{i n \alpha} \\
\rho_{-n} & =\rho_{n}^{\text {c.c. }} .
\end{aligned}
$$

Here $d \phi=d s / R$, where $s$ is the particle position along the ring and $2 \pi R$ is the ring circumference. The average effect of the beam-beam interaction depends on the beam-beam parameter $\xi_{\text {Вв }}$ which is usually small, $\xi_{\text {Вв }}<$ 0.05 . Hence, the steady-state effect of the beam-beam force leads only to small transverse potential well distortion and cannot explain the beam blowup. This also allows us to use Gaussian distribution as the zero-order bunch distribution function.

The Hamiltonian of the system is the sum of the Hamiltonians of two beams

$$
H=H_{1}\left(I_{1}, \alpha_{1}, \phi\right)+H_{2}\left(I_{2}, \alpha_{2}, \phi\right) .
$$

Each one of them depends on the distribution function of another bunch. The Hamiltonian in the angle-action variables takes into account all azimuthal harmonics of the distribution function $\rho_{k}, H_{1}(I, \alpha, \phi)=H_{H}^{(1)}+\Delta H_{1}$, 


$$
\begin{aligned}
\Delta H_{1}(I, \alpha, \phi)= & 2 \pi \Lambda_{1} \sum_{n, m \neq 0} e^{i(n \alpha-m \phi)} \int d I^{\prime}\left[S_{n 0}\left(I, I^{\prime}, \Delta y\right) \rho_{H}^{(2)}\left(I^{\prime}\right)\right. \\
& \left.+\sum_{k} S_{n, k} \rho_{k}^{(2)}\left(I^{\prime}\right)\right]+2 \pi \Lambda_{1} \sum_{n} e^{i n \alpha} \sum_{k} \int d I^{\prime} S_{n, k}\left(I, I^{\prime}, \Delta y\right) \rho_{k}^{(2)}\left(I^{\prime}, \phi\right) .
\end{aligned}
$$

Here,

$$
\Lambda_{1}=\frac{2 N_{b}^{(2)} r_{0}}{2 \pi \gamma_{1} \epsilon_{y}^{(1)}} .
$$

Function $S_{n, k}$ is obtained directly from the kick due to Coulomb interaction between bunches averaged over the Gaussian distribution in the $x$ plane. The numeric factor in $S_{n, k}$ for the first beam is slightly different $(1 / \sqrt{2}$ or $\sqrt{\pi / 2}$ ) depending on whether the averaging is carried out for both beams or only over the $x$ distribution of the fist beam. This difference does not affect the following consideration. Assuming equal horizontal rms dimensions $\sigma_{x}$ of two beams at the interaction point (IP) and neglecting anharmonic corrections to the particle trajectories, we write the function $S_{n, l}$ for a flat beam as

$$
S_{n, l}\left(I, I^{\prime}, \Delta y_{0}\right)=-\int_{0}^{\infty} \frac{d x}{x} \operatorname{erfc}\left[x \sqrt{2} \frac{\sigma_{x}}{\sigma_{2, y}}\right]\left\{\cos \left[(n-l) \frac{\pi}{2}+\frac{\Delta y_{0} \sqrt{2}}{\sigma_{2, y}} x\right] J_{n}\left(\frac{2 \sigma_{1, y}}{\sigma_{2, y}} \sqrt{I} x\right) J_{l}\left(2 \sqrt{I^{\prime}} x\right)-1\right\},
$$

where $\operatorname{erfc}(\xi)=e^{\xi^{2}}[1-\operatorname{erf}(\xi)], \operatorname{erf}(\xi)$ is the error function. Here we dropped the factor $e^{-x^{2} / 4 \sigma_{x}^{2}} \simeq 1$ for typical $|x|<\sigma_{x}$.

For a flat beam, the main contribution is given by $\xi=x \sigma_{x} / \sigma_{y} \gg 1$. In this case, $\operatorname{erfc}(\xi) \simeq 1 /(\xi \sqrt{\pi})$. For more details, see [7].

The steady-state Hamiltonian $H_{H}^{(1)}(I)=Q_{1} I-\Delta H_{H}^{(1)}$, where $Q_{1}$ is the zero-current tune. The second term, $\Delta H_{H}(I)$, takes into account the average part of the beam-beam potential defined by the contribution of $\rho_{H}$, which is not included in Eq. (3),

$$
\Delta H_{H}^{(1)}(I)=-\frac{\Lambda_{1} \sigma_{2 y}}{\sqrt{2 \pi} \sigma_{x}} \int_{0}^{\infty} \frac{d}{x^{2}}\left\{J_{0}\left(\frac{2 \sigma_{1, y} \sqrt{I}}{\sigma_{2, y}} x\right) \cos \left[\frac{\Delta y_{0} \sqrt{2}}{\sigma_{2, y}} x\right] e^{-x^{2}}-1\right\} .
$$

Equation (6) defines frequency $\omega_{1}(I)=d H_{H}^{(1)} / d I=$ $Q_{1}+\Delta Q_{1}(I)$, where the beam-beam tune shift for the first beam (averaged over distribution in the $x$ plane) is

$$
\begin{aligned}
\Delta Q_{1}(I)= & \frac{\Lambda_{1} \sigma_{1, y}}{\sigma_{x} \sqrt{2 \pi I}} \\
& \times \int_{0}^{\infty} \frac{d x}{x} J_{1}\left(\frac{2 \sigma_{1, y} \sqrt{I}}{\sigma_{2, y}} x\right) \cos \left(\frac{\Delta y_{0} \sqrt{2}}{\sigma_{2, y}} x\right) e^{-x^{2}} .
\end{aligned}
$$

For small amplitudes $I$ and $\Delta y_{0}=0, \delta Q(I)$ is proportional to the beam-beam parameter $\xi_{\mathrm{BB}}$. The extra factor $1 / \sqrt{2}$ comes from averaging over the $x$ distribution.

Hamiltonian $\mathrm{H}_{2}$ for the second beam can be obtained by interchanging indices 1 and 2 , and substituting $S_{k, n}\left(I^{\prime}, I, \Delta y\right)$ for $S_{n, k}\left(I, I^{\prime}, \Delta y\right)$.

\section{EFFECT OF THE NONLINEAR RESONANCES}

If nonzero components $\rho_{k}$ can be neglected, the simplified Hamiltonian is

$$
H_{1}(I, \alpha, \phi)=H_{H}^{(1)}+\sum_{n} \sum_{m \neq 0} U_{n}(I) e^{i(n \alpha-m \phi)},
$$

where the functions

$$
U_{n}(I)=2 \pi \Lambda_{1} \int d I^{\prime} S_{n 0}\left(I, I^{\prime}, \Delta y\right) \rho_{H}^{(2)}\left(I^{\prime}\right)
$$

are

$$
\begin{aligned}
U_{n}(I)= & -\frac{\Lambda_{1} \sigma_{y}}{\sigma_{x} \sqrt{2 \pi}} \\
& \times \int_{0}^{\infty} \frac{d x}{x^{2}} e^{-x^{2}} J_{n}\left[2 x \frac{\sigma_{1, y}}{\sigma_{2, y}} \sqrt{I}\right] \cos \left[\psi_{n}(x)\right] .
\end{aligned}
$$

Here, $\psi_{n}(x)=n \pi / 2+\left(\Delta y_{0} / \sigma_{2 y}\right) \sqrt{2} x$. For zero offsets $U_{n} \neq 0$ only for even $n=2 k$. In this case, $U_{2 k}$ is given in terms of the degenerate hypergeometric function,

$$
\begin{aligned}
U_{2 k}= & (-1)^{k+1} \frac{\Lambda_{1} \sigma_{2 y}}{2 \sigma_{x} \sqrt{2 \pi}} \frac{\Gamma(k-1 / 2)}{\Gamma(2 k+1)}\left(\frac{\sigma_{y 1}}{\sigma_{y 2}}\right)^{2 k}\left(\frac{I}{\epsilon_{1}}\right)^{k} \\
& \times F\left[k-1 / 2,2 k+1,-\left(\frac{\sigma_{y 1}}{\sigma_{y 2}}\right)^{2} \frac{I}{\epsilon_{1}}\right] .
\end{aligned}
$$

Hamiltonian Eq. (8) is the Hamiltonian of a nonlinear oscillator with frequency $\omega_{1}(I)=d H_{H}(I) / d I$ in the external periodic potential.

The perturbation due to beam-beam kicks is small, of the order of $\xi_{\mathrm{BB}}$, except for $I$ near the resonances $\omega_{1}\left(I_{R}\right)=m / n$. Particles with amplitudes $I \simeq I_{R}$ are trapped in a separatrix and the modulation of their amplitudes is of the order of the size of the separatrix, $\Delta I=$ $2 \sqrt{2 U_{n m}\left(I_{R}\right) / \omega_{1}^{\prime}\left(I_{R}\right)}$, where $\omega_{1}^{\prime}(I)=d^{2} H_{1}(I) / d I^{2}$. Usually, $\omega^{\prime}$ due to lattice nonlinearities is small. In this case, both terms $U_{n, m}$ and $\omega^{\prime}$ are proportional to $\Lambda$ and dependence of the width $\Delta I$ on current arises only through the dependence of the shape $\rho_{H}$ on current. For $\Delta I$ of the 


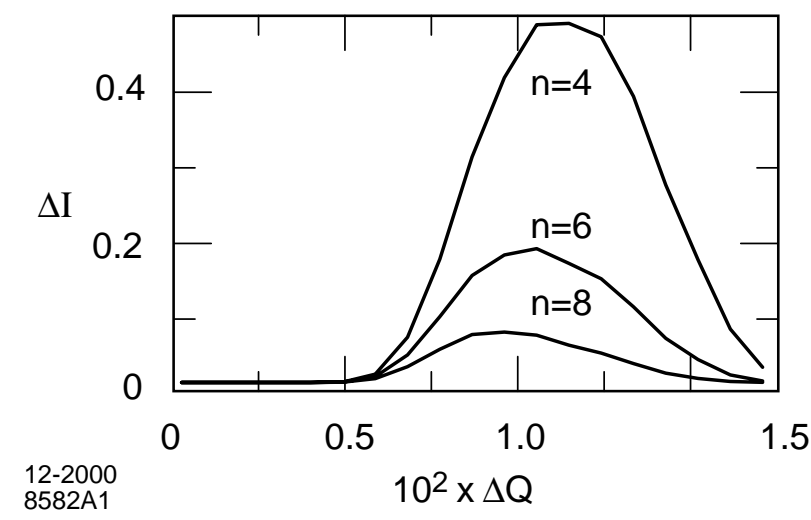

FIG. 1. Distortion of the beam emittance $\Delta I$ due to $n=2 k$-order resonance vs detuning from the resonance.

order of 1, the rms $\sigma_{y}$ defining $U_{n, m}$ has to be understood as a self-consistent parameter.

For small $I$ and zero offsets, the width of an even resonance $n=2 k$ is

$$
(\Delta I)_{2 k}=\frac{4 \sqrt{2}}{\pi^{1 / 4} 2^{k} \sqrt{(k-1 / 2) k !}} I_{R}^{k / 2}\left(\frac{\sigma_{y 1}}{\sigma_{y 2}}\right)^{k-2} .
$$

The width is small for higher order $k \gg 1$ resonances.

Particles trapped in the resonance separatrix change the distribution function [8]. For small separatrices

$$
\rho(I)=\frac{1}{Z} e^{-(1 / T)\left[H(I) \mp \omega\left(I_{R}\right) \Delta I \Psi(0)\right]},
$$

where $H(I) \simeq Q_{y} I, T=Q_{y} \epsilon_{y}, \Psi(0)=0.69$, and the plus (minus) sign corresponds to amplitudes above (below) resonance amplitude $I_{R}$.

Resonance changes unperturbed $\mathrm{rms}\langle I\rangle=1$ to

$$
\langle I\rangle=1+2(\Delta I)_{2 k} \Psi(0) I_{R} e^{-I_{R}},
$$

for small $(\Delta I)_{n}$.

Emittance distortion is small for higher order resonances; see Fig. 1.

\section{THE FOKKER-PLANCK EQUATION}

In this section we assume that the weak-strong resonances $\omega=n / m$ considered in the previous section are avoided by a proper choice of the working point in the tune diagram. This allows us to drop the term proportional to $\rho_{H}$. Perturbation in this case is produced by the azimuthal harmonics of the distribution function, $H_{1}(I, \alpha, \phi)=$ $H_{H}^{(1)}+\Delta H_{1}$,

$$
\begin{aligned}
\Delta H_{1}^{(1)}= & 2 \pi \Lambda_{1} \sum_{n, m=-\infty}^{\infty} e^{i(n \alpha-m \phi)} \\
& \times \int d I^{\prime} \sum_{k=-\infty}^{\infty} S_{n, k} \rho_{k}^{(2)}\left(I^{\prime}, \phi\right) .
\end{aligned}
$$

Term $k=0$ describes dynamic variation of the distribution function and plays a crucial role in the following considerations.

Let us start with the Fokker-Planck equation in $y, q=$ $d y / d s$, and $\phi$ variables:

$$
\frac{\partial \rho}{\partial \phi}+\{H, \rho\}_{q, y}=\gamma_{0} \frac{\partial}{\partial q}\left[\frac{D}{\gamma_{0}} \frac{\partial \rho}{\partial q}+q \rho\right] .
$$

Here the diffusion $D$ is due to fluctuations of the synchrotron radiation (SR) in the horizontal plane and $(x-y)$ coupling, $\gamma_{0}$ is damping in the vertical plane. Damping gives $\langle q\rangle \propto e^{-\gamma_{0} s / R}$, diffusion causes rms growth $\left\langle q^{2}\right\rangle=$ $2 D s / R$, and their ratio defines equilibrium temperature $T=\left\langle q^{2}\right\rangle=D / \gamma_{0}=\epsilon_{y} / \beta_{y}$ and the vertical emittance $\epsilon_{y}$.

The right-hand side (RHS) of Eq. (16) averaged over $\alpha$ can be written in $I, \alpha$ variables as

$$
R_{k}^{(1)}=\gamma_{0} \frac{\partial}{\partial I}\left\{I \frac{\partial \rho_{k}^{(1)}}{\partial I}+I \rho_{k}^{(1)}\right\}-\gamma_{0} \frac{k^{2}}{4 I} \rho_{k}^{(1)} .
$$

Here we have neglected betatron resonances $Q_{y}=n / m$ considered above, replaced $Q_{y} \beta_{y}$ by its average value over one turn $\int(d \phi / 2 \pi) Q_{y} \beta_{y}=R$, and introduced dimensionless $\gamma_{0}=1 /\left(\omega_{0} \tau_{\mathrm{SR}}\right)$, where $\omega_{0} /(2 \pi)$ is revolution frequency and $\tau_{\mathrm{SR}}$ is radiation damping time.

The Fokker-Planck equation for azimuthal harmonics $\rho_{k}(I, \phi)$ takes the form

$$
\begin{array}{rl}
\frac{\partial \rho_{k}^{(1)}}{\partial \phi}+i k \omega_{1}(I) \rho_{k}^{(1)}+i & i \Lambda_{1} \sum_{m, n, l}(k-l) \rho_{k-l}^{(1)} e^{i m \phi} \int d I^{\prime} d \alpha^{\prime} \rho_{n}^{(2)}\left(I^{\prime}\right) \frac{\partial S_{l n}\left(I, I^{\prime}\right)}{\partial I}- \\
& i \Lambda_{1} \sum_{m, n, l} l \frac{\partial\left(\rho_{H} \delta_{k, l}+\rho_{k-l}^{(1)}\right)}{d I} e^{i m \phi} \int d I^{\prime} d \alpha^{\prime} \rho_{n}^{(2)}\left(I^{\prime}\right) S_{l n}=R_{k}^{(1)} .
\end{array}
$$

Harmonics $\rho_{0}$ satisfy the following equation:

$$
\frac{\partial \rho_{0}^{(1)}}{\partial \phi}-i \Lambda_{1} \frac{\partial}{\partial I} \sum_{m, n, l} l\left[\rho_{l}^{(1)}\right]^{c . c c} e^{i m \phi} \int d I^{\prime} d \alpha^{\prime} \rho_{m}^{(2)}\left(I^{\prime}\right) S_{l m}\left(I, I^{\prime}, \Delta y_{0}\right)=R_{0}^{(1)} .
$$

The equation for the second beam can be obtained by replacing index $1 \rightarrow 2$ and $S_{n l}\left(I, I, \Delta y_{0}\right)$ by $S_{l, n}\left(I^{\prime}, I, \Delta y_{0}\right)$ because $S_{l n}\left(I, I^{\prime},-\Delta y_{0}\right)=S_{n l}\left(I^{\prime}, I, \Delta y_{0}\right)$.

For small coherent tune shifts $\Delta Q \ll 1$, dependence on $\phi$ of azimuthal harmonics is given mainly by the factor $e^{-i k Q_{y} \phi}$. 
Generally, there is a dense net of resonances in the plane $Q_{y 1}, Q_{y 2}$. The width of the resonance line is of the order of the beam-beam parameter and increases with the bunch current. Eventually, resonances overlap and the motion becomes stochastic.

Let us consider bunch current slightly above the threshold of the coherent beam-beam instability. In this case, the tune spread with amplitude within the bunch distribution can be smaller than the distance to the low order resonances. Higher order resonances are suppressed by the synchrotron damping. For electron machines it is sufficient to consider resonances of the order of $\left|n_{0}\right|+$ $\left|m_{0}\right|<=4$. In this case, we can consider isolated reso- nances and assume that beam dynamics is defined by the resonance $\left(n_{0}, l_{0}\right)$ with the smallest detuning $\Delta m \equiv$ $n_{0} Q_{1, y}-l_{0} Q_{2, y}-m_{0}$.

This allows us to average fast oscillating terms and retain only equations for harmonics $\rho_{n 0}^{(1)}$ and $\rho_{l 0}^{(2)}$.

Let us introduce slow functions $f_{n 0}^{(1)}$ and $f_{l 0}^{(2)}$,

$$
\begin{aligned}
& \rho_{n 0}^{(1)}=f_{1} e^{-i n_{0} Q_{1} \phi+i \Delta m / 2 \phi}, \\
& \rho_{l 0}^{(2)}=f_{2} e^{-i l_{0} Q_{2} \phi-i \Delta m / 2 \phi} .
\end{aligned}
$$

In the linear approximation, these functions satisfy the coupled system of equations

$$
\begin{aligned}
& \frac{\partial f_{1}}{\partial \phi}+i\left[n_{0}\left(\omega_{1}(I)-Q_{1}\right)+\frac{\Delta m}{2}\right] f_{1}-i \Lambda_{1} n_{0} \frac{\partial \rho_{H}^{(1)}}{\partial I} \int d I^{\prime} d \alpha^{\prime} f_{2}\left(I^{\prime}\right) S_{n 0, l 0}\left(I, I^{\prime}\right)=0, \\
& \frac{\partial f_{2}}{\partial \phi}+i\left[l_{0}\left(\omega_{2}(I)-Q_{2}\right)-\frac{\Delta m}{2}\right] f_{2}-i \Lambda_{2} l_{0} \frac{\partial \rho_{H}^{(2)}}{\partial I} \int d I^{\prime} d \alpha^{\prime} f_{1}\left(I^{\prime}\right) S_{n 0, l 0}\left(I^{\prime}, I\right)=0 .
\end{aligned}
$$

The system has a solution in the form

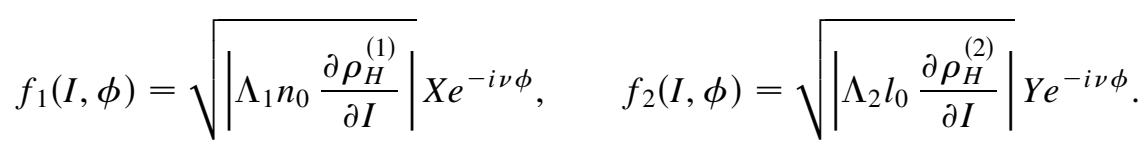

The vectors $(X, Y)$ are eigenvectors of the matrix

$$
\left(\begin{array}{cc}
\left(n_{0} \Delta Q_{1}+\Delta m / 2\right) \delta\left(I-I^{\prime}\right) & -\operatorname{sgn}\left(n_{0}\right) K\left(I, I^{\prime}\right) \\
-\operatorname{sgn}\left(l_{0}\right) K^{T}\left(I, I^{\prime}\right) & \left(l_{0} \Delta Q_{2}-\Delta m / 2\right) \delta\left(I-I^{\prime}\right)
\end{array}\right) .
$$

Here $K\left(I, I^{\prime}\right)=2 \pi \sqrt{\left|\Lambda_{1} \Lambda_{2} n_{0} l_{0} \frac{\partial \rho^{(1)}}{\partial I} \frac{\partial \rho^{(2)}}{\partial I}\right|}$, and $K^{T}$ is the transposed matrix $K^{T}\left(I, I^{\prime}\right)=K\left(I^{\prime}, I\right)$. Because this matrix is real and symmetric, the eigenvalues $\nu$ are real and the system is stable provided $n_{0} l_{0}>0$. Hence, instability is possible only if $n_{0} l_{0}<0$, i.e., for the sum resonances.

It should be mentioned though that this conclusion is derived from the explicit form of $S_{n, l}\left(I, I^{\prime}\right)$ which was calculated by neglecting all anharmonic terms in the trajectory $Y(I, \alpha, \phi)$. It is well known that, for the microwave instability, exactly these terms are responsible for the onset of instability. However, for the beam-beam interaction at the sum resonances these terms give only small corrections although they may be important for the difference resonances.

It is worth noting that if $n-l=$ odd, $S_{n l} \neq 0$ only for nonzero offsets $\Delta y_{0}$.

The effect of a resonance on beam dynamics depends on the resonance order $\left|n_{0}\right|+\left|l_{0}\right|$ and the detuning $\Delta m=$ $n_{0} Q_{1, y}-l_{0} Q_{2 y}$. For the sum resonances $n_{0} l_{0}<0$, which are above and to the right of the working point, the tune is shifted toward the resonance due to finite amplitudes of betatron oscillations.

\section{THE LINEAR APPROXIMATION}

In the linear approximation, let us expand slow functions in Eqs. (21) and (22) over eigenvectors $V_{\nu}=\left\{Y_{\nu}, X_{\nu}\right\}$,

$$
\begin{aligned}
& f_{1}(I, \phi)=\frac{\partial \rho_{H}^{(1)}}{\partial I} \sum_{\nu} A_{\nu} Y_{\nu} e^{-i \nu \phi} \\
& f_{2}(I, \phi)=\frac{\partial \rho_{H}^{(2)}}{\partial I} \sum_{\nu} A_{\nu} X_{\nu} e^{-i \nu \phi} .
\end{aligned}
$$

Vectors $V_{\nu}(I)=\left(X_{\nu}, Y_{\nu}\right)$ are the eigenvectors (radial modes) of the matrix $M, \int M\left(I, I^{\prime}\right) V_{\nu}\left(I^{\prime}\right) d I^{\prime}=-\nu V_{\nu}(I)$,

$$
M=\left(\begin{array}{cc}
\left(-l_{0} \Delta Q_{2}+\Delta m / 2\right) \delta_{I I^{\prime}} & M_{2} \\
M_{1} & \left(-n_{0} \Delta Q_{1}-\Delta m / 2\right) \delta_{I I^{\prime}}
\end{array}\right),
$$




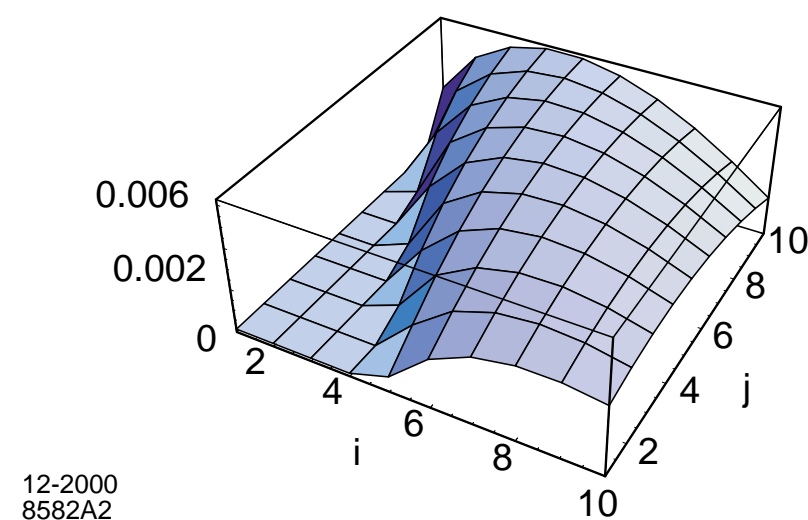

FIG. 2. (Color) The fastest growth rate of the radial modes for the resonance $n=2, l=-1$ vs beam currents. Bunch population $(N / N 0)_{\mathrm{LER}}=1+0.2(i-1),(N / N 0)_{\mathrm{HER}}=1+$ $0.2(j-1),(i, j)=1, \ldots, 10 ; Q_{y}$ LER $=34.64 ; Q_{y}$ HER $=$ 23.64.

where $\delta_{I I^{\prime}}=\delta\left(I-I^{\prime}\right)$,

$$
\begin{aligned}
& M_{1}\left(I, I^{\prime}\right)=2 \pi \Lambda_{1} n_{0} \frac{d \rho_{H}^{(2)}\left(I^{\prime}\right)}{d I^{\prime}} S_{n 0, l 0}\left(I, I^{\prime}\right), \\
& M_{2}\left(I, I^{\prime}\right)=2 \pi \Lambda_{2} l_{0} \frac{d \rho_{H}^{(1)}\left(I^{\prime}\right)}{d I^{\prime}} S_{n 0, l 0}\left(I^{\prime}, I\right) .
\end{aligned}
$$

The norm $\int d I \hat{V}_{\mu} V_{\nu}=\delta_{\nu, \mu}$, where $\hat{V}_{\mu}$ are eigenvectors of the transposed matrix $M^{T}$.

The nominal parameters of the B Factory are [LER/HER stand for the low energy (positron) and high energy (electron) rings, respectively] $N_{\mathrm{HER}}^{0}=1.74 \times 10^{10}, N_{\mathrm{LER}}^{0}=$ $5.617 \times 10^{10}, \quad \sigma_{x}=145.4 \mu \mathrm{m}, \quad \sigma_{y, \mathrm{LER}}=\sigma_{Y, \mathrm{HER}}=$ $3.79 \mu \mathrm{m}, \quad\left(\omega_{0} \tau_{d}\right)^{-1}=2.92 \times 10^{-5}$, and the values $\Lambda_{1}=1.988, \Lambda_{2}=1.782$ have been used for numerical calculations unless specified otherwise.

At low bunch currents all eigenvalues are real. At a threshold current, one of the modes becomes linearly unstable; see Fig. 2.

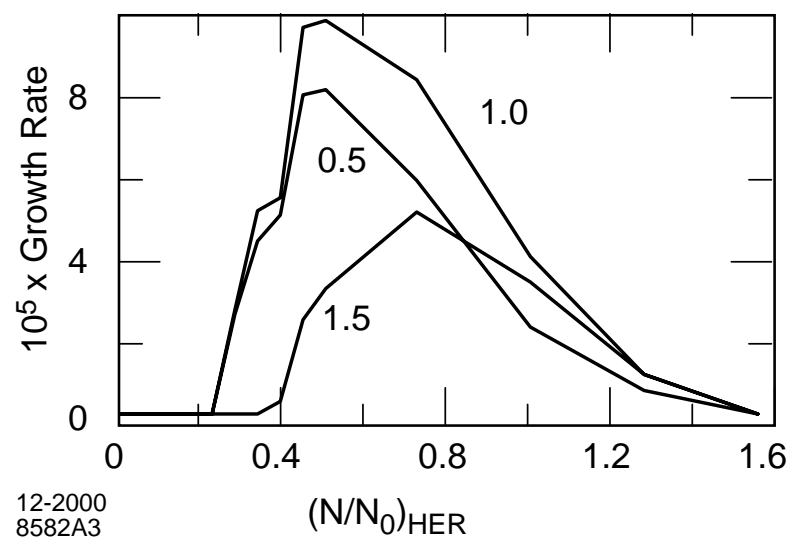

FIG. 3. The maximum growth rate of radial modes for the resonance $n=2, l=-3 . \quad Q_{y} \mathrm{LER}=34.57, Q_{y}$ HER $=$ 23.62, nominal bunch population. Beam offset is shown in units of $\sigma_{y}$.

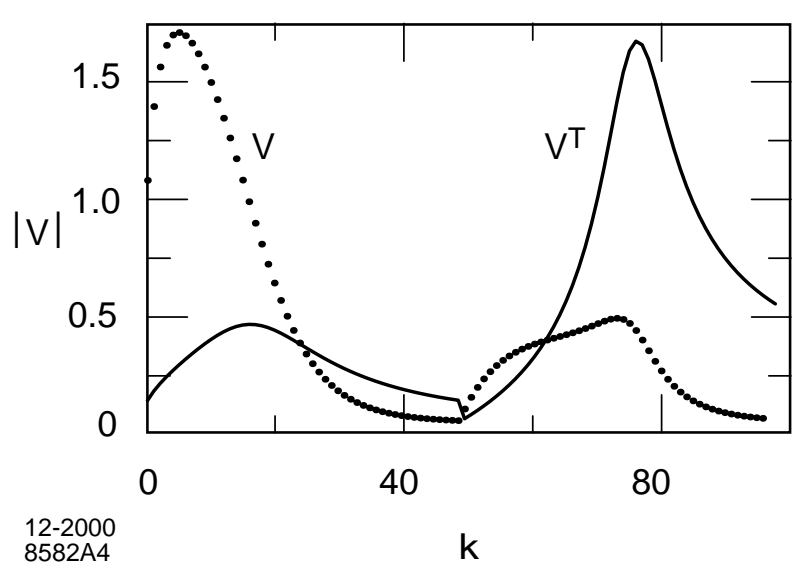

FIG. 4. Eigenvectors $V(I)$ and $\hat{V}(I), I=0.05(k-1)$, of the most unstable radial mode vs $k=1, \ldots, 100$. The left part of the curves $k=1, \ldots, 50$ corresponds to vector $X$, the right part to $Y$.

The threshold current depends on the detuning from the closest resonance and the beam offset $\Delta y$. Figure 3 shows the maximum growth rate of radial modes for the resonance $n_{0}=2, l_{0}=-3$ vs $\left(N / N_{0}\right)_{\mathrm{HER}}$ for the three offset $\Delta y_{0}$. The latter is given in units of the vertical rms $\Delta y_{0} / \sigma_{2}=0.1+0.2(j-1), j=1, \ldots, 10$.

At the nominal bunch current there is only one linearly unstable radial mode. The matrix $M\left(I, I^{\prime}\right)$, eigenvectors $V_{\nu}(I)$, and the functions $f_{1,2}$ were discretized on the mesh $i, j=100 \times 100$. It is worth noting that the number of radial modes depends on the rank of the matrix $M$, i.e., on the discretization step $\Delta I$, and does not have physical meaning. However, only a few of the modes are real coherent modes while others can be considered as singleparticle modes. The first can be defined as modes whose widths are larger than $\Delta I$. Such modes have the lowest maximum magnitude of the normalized eigenvector $|V|^{2}$.

Figure 4 gives an example of the mode structure, i.e., $|V|^{2}$ and $|\hat{V}|^{2}$ of the most unstable mode. The eigenvectors of the most linearly stable mode (the mode with eigenvalue which is complex conjugated to the eigenvalue of the unstable mode) has the same structure. Nominal currents were used in calculations, $Q y_{\mathrm{HER}}=23.64$; $Q y_{\mathrm{LER}}=34.61$. The resonance $n_{0}=2, l_{0}=-1 \mathrm{de}-$ tuned by $\Delta Q=-0.03$ was used as an example. Maximum linear growth rate in this case is for the mode 62 , $\nu=0.00479+0.0011 i$, mode 61 is the most stable mode $\left(\mu=\nu^{*}\right)$. The SR decrements were set to be equal to $\gamma_{0}$.

\section{INTERACTION OF RADIAL MODES}

Consider a resonance with azimuthal numbers $n_{0}, l_{0}$ neglecting all other azimuthal modes. At the threshold of instability, one of the radial modes $V_{\nu}$ becomes unstable. Time evolution of the mode depends on the interaction of this mode with linearly stable radial modes of the same azimuthal mode. This is true if the coherent tune shift of the radial modes is small compared to the distance to other 
linear resonances. The validity of this assumption depends on the choice of the working point in the tune diagram but usually appears naturally for the moderate beam-beam parameters.

It is convenient to describe mode interaction expanding azimuthal harmonics of the distribution function in radial eigenmodes of the linear approximation,

$$
\begin{aligned}
& \rho_{n 0}^{(1)}=\frac{\partial \rho_{H}^{(1)}}{d I} e^{-i n_{0} Q_{1} \phi+i \Delta m / 2 \phi} \sum_{\mu} A_{\mu} Y^{\mu}, \\
& \rho_{n 0}^{(2)}=\frac{\partial \rho_{H}^{(2)}}{d I} e^{-i l_{0} Q_{2} \phi-i(\Delta m / 2) \phi} \sum_{\mu} A_{\mu} X^{\mu},
\end{aligned}
$$

where $X_{\mu}$ and $Y_{\mu}$ are components of the vector $V_{\mu}=$ $\left(X_{\mu}, Y_{\mu}\right)$ of the matrix $M$, Eq. (25), with the eigenvalue $\mu$.

We assume that $V_{\mu}$ are normalized, $\int d I \hat{V}_{\mu} V_{\nu}=\delta_{\nu, \mu}$, where $\hat{V}_{\mu}$ are eigenvectors of the transposed matrix $M^{T}$ with the same eigenvalue as vector $V_{\mu}$.

Let us neglect all azimuthal modes $\rho_{k}(I, \phi)$ except modes in the resonance pair $\left(n_{0}, l_{0}\right)$, and take into account only two radial modes: the linearly unstable mode $V_{\nu}$ and a linearly stable mode $V_{\mu}$. The choice of $V_{\mu}$ will be specified later. To simplify notation, we denote their amplitudes as $A_{k}, k=1$ for unstable mode $\nu$, and $k=2$ for stable mode with eigenvalue $\mu$. The amplitudes satisfy the system of two coupled equations

$$
\frac{\partial A_{k}}{\partial \phi}+\left(i \lambda_{k}+\gamma_{k}\right) A_{k}=i \sum_{l=1}^{2} d_{k, l} A_{l},
$$

where $\lambda_{1}=\nu, \lambda_{2}=\mu$. The SR decrement $\gamma_{k}$ is given by

$$
\gamma_{k} A_{k}=\int d I\left[\frac{\hat{X}_{k} R_{l 0}^{(2)}}{\left[\rho_{h}^{(2)}\right]^{\prime}} e^{i\left(l_{0} Q_{2}+\Delta m / 2\right) \phi}+\frac{\hat{Y}_{k} R_{n 0}^{(1)}}{\left[\rho_{h}^{(1)}\right]^{\prime}} e^{i\left(n_{0} Q_{1}-\Delta m / 2\right) \phi}\right],
$$

where $R^{(1,2)}$ are given by Eq. (17). Here we used notation $[\rho]^{\prime}=d \rho / d I$. The RHS is of the order of SR decrement $\gamma_{0}$ and small. This allows us to neglect mode coupling due to the effect of the SR. In this case, Eq. (30) defines SR mode decrements. It is interesting to notice that the SR decrement for the unstable mode is minimal.

The coefficients $d_{k, l}$ describe mode coupling due to perturbation of the zero harmonics $\rho_{0}$ of the distribution function,

$$
\begin{aligned}
d_{k, l}= & \int d I \hat{X}_{k}(I)\left\{\left(-\lambda_{l}+l_{0} \Delta Q_{2}-\frac{\Delta m}{2}\right) X_{l} \frac{\left[\rho_{0}^{(2)}\right]^{\prime}}{\left[\rho_{H}^{(2)}\right]^{\prime}}-2 \pi \Lambda_{2} l_{0} X_{l} \int d I \rho_{0}^{(1)}(I) \frac{d S_{00}(I, I)}{d I}\right\} \\
& +\int d I \hat{Y}_{k}(I)\left\{\left(-\lambda_{l}+n_{0} \Delta Q_{1}+\frac{\Delta m}{2}\right) Y_{l} \frac{\left[\rho^{(1)}\right]^{\prime}}{\left[\rho_{H}^{(1)}\right]^{\prime}}-2 \pi \Lambda_{1} n_{0} Y_{l} \int d I \rho_{0}^{(2)}(I) \frac{d S_{00}(I, I)}{d I}\right\} .
\end{aligned}
$$

Time variation of these coefficients can be obtained from Eq. (19),

$$
\frac{\partial d_{k l}}{\partial \phi}+\gamma_{0} d_{k l}=i \sum_{k^{\prime}, l^{\prime}} P_{k^{\prime}, l^{\prime}}^{k, l}\left[A_{k^{\prime}}\right]^{\text {c.c. }} A_{l^{\prime}},
$$

where parameters $P_{k^{\prime} l^{\prime}}^{k l}$ are

$$
\begin{aligned}
P_{k^{\prime} l^{\prime}}^{k, l}= & -\int d I \frac{d}{d I}\left[\left(-\lambda_{l}+l_{0} \Delta Q_{2}-\frac{\Delta m}{2}\right) \frac{\hat{X}_{k}(I) X_{l}}{\left[\rho_{H}^{(2)}\right]^{\prime}}\right] \frac{d}{d I}\left[\left(\lambda_{k^{\prime}}^{\mathrm{c} . c .}-\lambda_{l^{\prime}}\right) X_{k^{\prime}}^{\mathrm{c} . c} \cdot X_{l^{\prime}}\left(\rho_{H}^{(2)}\right)^{\prime}\right] \\
& -\int d I \frac{d}{d I}\left[\left(-\lambda_{l}+n_{0} \Delta Q_{1}+\frac{\Delta m}{2}\right) \frac{\hat{Y}_{k}(I) Y_{l}}{\left[\rho_{H}^{(1)}\right]^{\prime}}\right] \frac{d}{d I}\left[\left(\lambda_{k^{\prime}}^{\text {c.c. }}-\lambda_{l^{\prime}}\right) Y_{k^{\prime}}^{\text {c.c. }} Y_{l^{\prime}}\left(\rho_{H}^{(1)}\right)^{\prime}\right]
\end{aligned}
$$

Terms containing $S_{00}$ are not written here. Their contribution is small.

\section{SINGLE MODE IN THE NONLINEAR REGIME}

The dynamics of the unstable mode is defined by perturbation of the distribution function $\rho_{0}$ by the growing mode. This perturbation changes the growth rate and may lead to the mode saturation. This concept corresponds to the quasilinear theory [9].

Let us consider first a simple case of a single unstable mode. In this case, there is only one amplitude $A=A_{1}$, $d=d_{11}, P=P_{1,1}^{1,1}, \gamma=\gamma_{1}$ and the system Eqs. (29) and (31) is reduced to

$$
\frac{d A}{d \phi}+(i \nu+\gamma) A=i d A, \quad \frac{\partial d(\phi)}{\partial \phi}+\gamma_{0} d=i P|A|^{2} .
$$

There is always a trivial solution $A=0$ corresponding to the linearly stable beam.

A nontrivial solution $\dot{d}=0, d=d_{0}, A=A_{0} e^{-i \Omega \phi}$, $\operatorname{Im}[\Omega]=0$ corresponds to an unstable mode which saturates at some amplitude $A_{0}$ constant in time. The momentum $d$ of $\rho_{0}$ is constant, which corresponds to a steady-state beam distortion. Such a distortion may contribute to the well-known flip-flop phenomenon when at least one of the beams is blown up. Equation (34) defines amplitudes $A_{0}, d_{0}$ and the coherent frequency shift $\Omega$, 


$$
\begin{gathered}
\left|A_{0}\right|^{2}=\gamma_{0} \frac{(\operatorname{Im}[\nu]-\gamma)}{\operatorname{Re}[P]}, \quad d_{0}=i \frac{P}{\gamma_{0}} A_{0}^{2}, \\
\Omega=\operatorname{Re}[\nu]+\frac{\operatorname{Im}[P]}{\operatorname{Re}[P]}(\operatorname{Im}[\nu]-\gamma) .
\end{gathered}
$$

For a mode to be unstable, $\operatorname{Im}[\nu]-\gamma$ has to be positive. Hence, solution Eq. (35) exists if $\operatorname{Re}[P]>0$. This condition is easily satisfied because $P$ is given by the second derivative of a function at the maximum; see Eq. (33).

The condition of stability of the nontrivial solution can be obtained by linearizing Eq. (34) $A=\left(A_{0}+a\right) e^{-i \Omega \phi}$, $d=d_{0}+c$ :

$$
\begin{aligned}
\frac{d a}{d \phi}+(i \nu+\gamma-i \Omega) a & =i\left(d_{0} a+A_{0} c\right), \\
\frac{d c(\phi)}{d \phi}+\gamma_{0} c & =i P A_{0}\left(a+a^{\text {c.c. }}\right) .
\end{aligned}
$$

By definition of the fixed point (FP) [cf. Eq. (34)], $i \nu+$ $\gamma-i \Omega=i d_{0}$. Define phase $\xi, P=|P| e^{i \xi}$, and introduce $g, b$, and $c=i g e^{i \xi}, a=b e^{i \xi}$. Then,

$$
\frac{d g}{d \phi}+\gamma_{0} g=2\left|A_{0}\right| \operatorname{Re}[P] b, \quad \frac{d b}{d \phi}+\left|A_{0}\right| g=0 .
$$

The eigenvalue $g, b \propto e^{i \zeta \phi}$ is

$$
\zeta=\frac{i \gamma_{0}}{2} \pm \sqrt{2 \gamma_{0}(\operatorname{Im} \nu-\gamma)-\left(\frac{\gamma_{0}}{2}\right)^{2}}
$$

Hence, the nontrivial solution is stable if $\operatorname{Im}[\nu]>\gamma$, whereas the linear solution becomes unstable. Figure 5 shows amplitude $A$ vs time for three initial values near the FP Eq. (35).

Harmonics $\rho_{n 0}^{(1)}$ and $\rho_{l 0}^{(2)}$ of the beam distribution oscillate and produce coherent signals at the frequencies

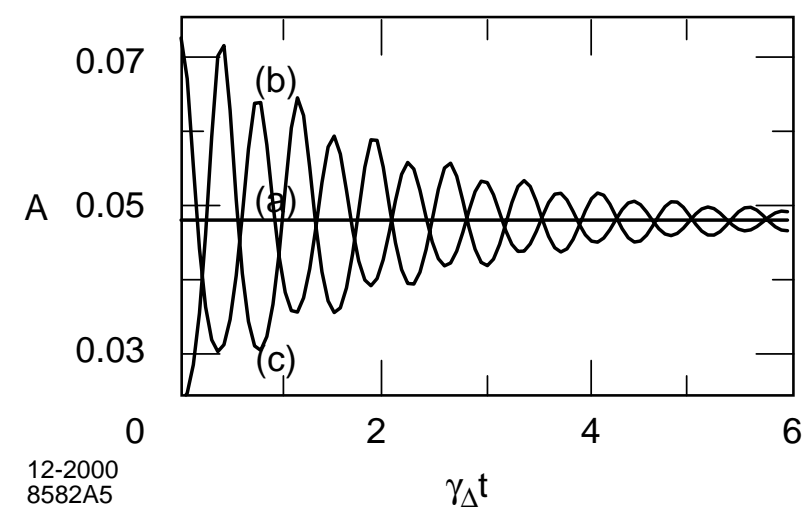

FIG. 5. Beam dynamics in the vicinity of the single mode FP. Resonance $n_{0}=1, l_{0}=-2$, linear growth rate $\operatorname{Im}[\nu]=$ 0.0025 , SR damping rate $\gamma_{d}=2.92 \times 10^{-5}$. Initial condition $d=d_{0}$ is given by Eq. (35). Three curves correspond to initial amplitudes (a) $A=A_{0}$, (b) $A=1.5 A_{0}$, and (c) $A=0.5 A_{0}$. $\omega_{1}=n_{0} Q_{1 y}-\Delta m_{0} / 2+\Omega$ and $\omega_{2}=l_{0} Q_{2 y}+\Delta m_{0} / 2-$ $\Omega$, correspondingly.

The nontrivial solution changes transverse beam emittance by $\Delta I=\int I d I d \alpha \rho_{0}(I, \alpha) . \Delta I$ can be found from Eq. (19) where we approximate the RHS $R_{0} \simeq-\gamma_{0} \rho_{0}$. This gives for the first beam

$$
\begin{aligned}
\frac{\partial}{\partial \phi} \Delta I_{1}+\gamma_{0} \Delta I_{1}= & -2 \operatorname{Im}[\nu]\left|A_{\nu}\right|^{2} \\
& \times \int d I d \alpha \frac{\partial \rho_{H}^{(1)}}{\partial I}\left|Y_{\nu}\right|^{2} .
\end{aligned}
$$

The blowup in the steady state is proportional to the increment $\operatorname{Im}[\nu]>0$ of the unstable mode

$$
\Delta I_{1}=\frac{2 \operatorname{Im}[\nu]}{\gamma_{0}}\left|A_{0}\right|^{2} \int d I e^{-I}\left|Y_{\nu}\right|^{2} .
$$

$A_{0}$ is defined in Eq. (35) and, hence, $\Delta I_{1}$ is independent of $\gamma_{0}$. The result for the second beam can be obtained by interchanging indices $(1,2)$ and replacing $Y_{\nu}$ by $X_{\nu}$.

In this section we have neglected the coupling of the unstable mode to other radial modes. In the next section we show that such an interaction can lead to excitation of the linearly stable modes and periodic oscillations of the amplitudes.

\section{TWO RADIAL MODES}

Next, we consider two interacting modes where only one of them is linearly unstable with the eigenvalue $\nu, \operatorname{Im}[\nu]>$ 0 . Initially, the dynamics of the system is dominated by the exponentially growing mode $A_{y}$. Equation (32) shows that $d_{i k}$ is driven by the term $\dot{d}_{i, k}=i P_{\nu, \nu}^{i k}\left|A_{\nu}\right|^{2}$. Let us neglect amplitudes of linearly stable modes and consider $d_{i k}$ as constant. Then amplitude $A_{\nu}$ of the unstable mode grows in time with dynamic increment $\Gamma_{\nu}=$ $\operatorname{Im}[\nu]-\gamma_{\nu}-\operatorname{Im}\left[d_{\nu \nu}\right]$. If $\operatorname{Re}\left[P_{\nu, \nu}^{\nu, \nu}\right]>0$, the dynamic increment goes at certain time to zero. The amplitude $A_{\nu}$ saturates at

$$
\left|A_{\nu}^{\mathrm{sat}}\right|^{2}=\gamma_{0} \frac{\operatorname{Im}[\nu]-\gamma_{\nu}}{\operatorname{Re}\left[P_{\nu \nu}^{\nu \nu}\right]} .
$$

From the equation for the linearly stable mode $A_{\mu}, \mu \neq \nu$, follows in the same way that $A_{\mu}$ varies in time with the dynamic increment $\Gamma_{\mu}=\operatorname{Im}[\mu]-\gamma_{\mu}-\operatorname{Im}\left[d_{\mu, \mu}\right]$. At saturation, $d_{\mu, \mu}=\left(i / \gamma_{0}\right) P_{\nu, \nu}^{\mu, \mu}\left|A_{\nu}^{\text {sat }}\right|^{2}$ and

$$
\Gamma_{\mu}^{\mathrm{sat}}=\operatorname{Im}[\mu]-\gamma_{\mu}-\left(\operatorname{Im}[\nu]-\gamma_{\nu}\right) \frac{\operatorname{Re}\left[P_{\nu \nu}^{\mu \mu}\right]}{\operatorname{Re}\left[P_{\nu \nu}^{\nu \nu}\right]} .
$$

If $\Gamma_{\mu}^{\text {sat }}>0$, the linearly stable mode $\mu$ becomes unstable while linearly unstable mode $\nu$ saturates. This allows us to choose the most important stable mode as a mode with maximum positive $\Gamma_{\mu}^{\text {sat }}$. Such a mode becomes unstable first and the amplitude $A_{\mu}$ may start to grow when the linearly unstable mode goes to saturation.

The criterion formulated for selection of the linearly stable mode shows that such a mode is the most stable mode in the linear approximation. Indeed, for such a mode 
$P_{\nu \nu}^{\mu \mu}=-\left[P_{\nu \nu}^{\nu \nu}\right]^{\text {c.c. }}$ and the ratio $\operatorname{Re}\left[P_{\nu \nu}^{\mu \mu}\right] / \operatorname{Re}\left[P_{\nu \nu}^{\nu \nu}\right]$ is negative and maximal.

In this case, analysis of the FP of the system is simplified. To simplify the notations, we change $\nu \rightarrow 1, \mu \rightarrow 2$. If mode 2 is the most stable mode in the linear approximation, then the eigenvectors $V_{1}$ and $V_{2}$ have the same pattern and coefficients $P_{l m}^{i k}$ have symmetries:

$$
P_{1,1}^{1,1}=-P_{2,2}^{1,1}=-\left[P_{1,1}^{2,2}\right]^{\text {c.c. }}=\left[P_{2,2}^{2,2}\right]^{\text {c.c. }},
$$

$$
P_{1,1}^{1,2}=-P_{2,2}^{1,2}=-\left[P_{1,1}^{2,1}\right]^{\text {c.c. }}=\left[P_{2,2}^{2,1}\right]^{\text {c.c. }} .
$$

That leaves only two parameters $p=P_{1,1}^{1,1} / \gamma_{0}$ and $q=$ $P_{1,1}^{1,2} / \gamma_{0}$. The FP solution $\partial d_{i k} / \partial \phi=0, A_{k} \propto e^{-i \Omega \phi}$ with real $\Omega$ is given by

$$
\begin{aligned}
d_{1,1} & =i p x, & d_{1,2} & =i q x, \\
d_{2,1} & =d_{1,2}^{\text {c.c. }}, & & d_{2,2}=d_{1,1}^{\text {c.c. }},
\end{aligned}
$$

The frequency shift $\Omega=\operatorname{Re}[\nu]+x \operatorname{Im}[p]$, and the parameter $x \equiv\left|A_{1}\right|^{2}-\left|A_{2}\right|^{2}$ is defined by

$$
x=\frac{1}{(\operatorname{Re}[p])^{2}-|q|^{2}}\left\{\operatorname{Im}(\nu) \operatorname{Re}[p] \pm \sqrt{(\operatorname{Im} \nu)^{2}|q|^{2}+\gamma^{2}(\operatorname{Re}[p])^{2}-|q|^{2}}\right\} .
$$

A solution exists if $x$ is real. Stability of the solution can be analyzed linearizing Eqs. (29) and (32).

Depending on parameters, interaction of two radial modes may lead to different beam behavior. If the amplitude at which a single linearly unstable mode saturates leaves the dynamic increment of linearly stable mode negative, the system goes to the single mode saturation regime described in the previous section. Otherwise, the linearly unstable mode excites the linearly stable mode before saturation and dynamics is defined by interaction of these two modes.

If in the system of two modes there are one or more fixed points then, again, there are two possibilities. In the first, both modes go to saturation with some, generally, nonequal amplitudes. Such a regime means that after a transient period a new line appears in the spectrum while the line corresponding to the initially unstable mode disappears. Another possibility is that the growing amplitude of linearly stable mode $A_{\mu}$ may change the dynamic increment of the mode $A_{\nu}$ and this mode starts to decay while, if $\operatorname{Re}\left[P_{\mu \mu}^{\mu \mu}\right]>0$, the amplitude $A_{\mu}$ saturates. The process can repeat itself and there will be periodic oscillations with energy exchange between modes. Such a mechanism is responsible for the onset of the sawtooth instability [10].

\section{CONCLUSION}

The effect of the coherent beam-beam instability on the transverse emittance is considered for flat beams. Beambeam coherent modes can be excited by periodic beambeam kicks. Two types of resonances are considered. The first type, $Q_{1,2}(I)=m / n$, is due to resonance harmonics of the kicks produced by the opposite rigid bunch in the steady state. This type leads to resonances of nonlinear oscillator under external periodic excitation. In this case, the rms emittance changes due to finite size of the separatrix of the resonance. Another type is due to the sum resonances $n Q_{1}-l Q_{2}=m,(n l)<0$, in the system of two coupled beams. In this case, there are linearly unstable modes for bunch currents above threshold which saturate due to distortion of the bunch distribution by the unstable mode. The saturation may occur either for a single mode or due to interaction of unstable radial mode with linearly stable radial mode. The result depends crucially on the SR damping of the radial modes. Results are illustrated numerically. For the parameters of the PEP-II B Factory, saturation occurs already at amplitudes equal to a small fraction of the rms $\sigma$. This is, probably, why coherent modes are so difficult to observe in experiments.

\section{ACKNOWLEDGMENT}

This work was supported by Department of Energy Contract No. DE-AC03-76SF00515.

[1] A. W. Chao, in Physics of High Energy Particle Accelerators, edited by Melvin Month, Per F. Dahl, and Margaret Dienes, AIP Conf. Proc. No. 127 (AIP, New York, 1985), and references therein.

[2] A. W. Chao and R. Ruth, Part. Accel. 16, 201 (1985).

[3] K. Hirata, KEK Report No. 90-25, 1990.

[4] K. Yokoya et al., Part. Accel. 27, 181 (1990).

[5] Y. Alexahin, in Proceedings of the 14th ICFA Beam Dynamics Workshop, Frascati, 1997 (Laboratory Nazionali di Frascati dell'INFN, Frascati, Italy, 1997).

[6] LBL Report No. LBL-PUB-5379, SLAC Report No. SLAC-418, 1993.

[7] S. Heifets, SLAC Report No. SLAC-PUB-8279, 1999.

[8] R. E. Meller, Ph.D. thesis, Cornell University, 1986.

[9] Yongho Chin and Kaoru Yokoya, Nucl. Instrum. Methods Phys. Res., Sect. A 226, 223-249 (1984).

[10] S. A. Heifets, in Workshop on Instabilities of High Intensity Hadron Beams in Rings, edited by T. Roser and S. Y. Zhang, AIP Conf. Proc. No. 496 (AIP, New York, 1999). 